\title{
ERRATUM
}

\section{Preface: High Energy Astrophysics}

\author{
Bing Zhang ${ }^{1,2, *}$, Peter Mészáros ${ }^{3, \dagger}$ \\ ${ }^{1}$ Kavli Institute for Astronomy and Astrophysics and Department of Astronomy, Peking University, China \\ ${ }^{2}$ Department of Physics and Astronomy, University of Nevada, Las Vegas, USA \\ ${ }^{3}$ Department of Astronomy and Astrophysics, Department of Physics, and Center for Particle and Gravitational Astrophysics, \\ Pennsylvania State University, University Park, PA 16802, USA \\ Corresponding authors.E-mail: *zhang@physics.unlv.edu, ${ }^{\dagger} n n p @ a s t r o . p s u . e d u$ \\ Received January 29, 2014
}

Erratum to: Front. Phys., 2013, 8(6): 605-608

DOI 10.1007/s11467-013-0351-7

One author's name is spelled wrong - "Peter Mésáros" should be replaced by "Peter Mészáros".

The online version of the original article can be founded at

http://dx.doi.org/10.1007/s11467-013-0351-7 